\title{
THE STRONG RELAXATION LIMIT OF THE MULTIDIMENSIONAL ISOTHERMAL EULER EQUATIONS
}

\author{
JEAN-FRANÇOIS COULOMBEL AND THIERRY GOUDON
}

\begin{abstract}
We construct global smooth solutions to the multidimensional isothermal Euler equations with a strong relaxation. When the relaxation time tends to zero, we show that the density converges towards the solution to the heat equation.
\end{abstract}

\section{INTRODUCTION}

We are interested in the behavior of solutions to the multidimensional isothermal Euler equations with a strong relaxation term:

$$
\left\{\begin{array}{l}
\partial_{t} \rho+\nabla_{x} \cdot \mathbf{m}=0, \\
\partial_{t} \mathbf{m}+\nabla_{x} \cdot\left(\frac{\mathbf{m} \otimes \mathbf{m}}{\rho}\right)+a^{2} \nabla_{x} \rho=-\frac{1}{\tau} \mathbf{m},
\end{array}\right.
$$

where $\left.\rho: \mathbb{R}^{+} \times \mathbb{R}^{d} \rightarrow\right] 0,+\infty\left[\right.$ is the density, $\mathbf{m}: \mathbb{R}^{+} \times \mathbb{R}^{d} \rightarrow \mathbb{R}^{d}$ is the momentum, $a>0$ is the speed of sound, and $0<\tau \ll 1$ is a (small) relaxation time. The system (11) is considered in the whole space $\mathbb{R}^{d}$, and we add initial data for $(\rho, \mathbf{m})$. In this paper, the density will always be bounded away from vacuum. The velocity of the fluid is $\mathbf{u}:=\mathbf{m} / \rho$. The aim of this paper is to construct global smooth solutions to (1) with initial data that are independent of the relaxation time $\tau$, and to show that, in an appropriate time scaling, the density converges towards the solution to the heat equation as $\tau$ tends to 0 . The sound speed $a$ is always kept constant.

In the one-dimensional case, the convergence of the solutions to (1) towards the solution to the heat equation has been proved in [3] for arbitrarily large initial data in $B V(\mathbb{R})$ that are bounded away from vacuum. In this case, one also obtains a rate of convergence in $L^{2}([0, T] \times \mathbb{R})$ for the density by using an appropriate stream function; see [3].

For fixed $\tau>0$, the existence of global smooth solutions to (1) follows from a result by Yong [10] (which is the analogue of [2] in the multidimensional framework). We refer the reader to [8] for the existence of global smooth solutions in the isentropic case. We also refer the reader to [6] for the derivation of the porous media equation as the limit of the isentropic Euler equations in one space dimension. In this paper, we show that the result of [10] can be made independent of the relaxation time $\tau$. This is due to the special structure of the system (11). (It

Received by the editors November 19, 2004.

2000 Mathematics Subject Classification. Primary 35L25; Secondary 35L65, 35L45, 76N15.

Key words and phrases. Gas dynamics, relaxation, global smooth solutions.

The research of the authors was supported by the EU financed network HYKE, HPRN-CT2002-00282

(C)2006 American Mathematical Society 637 
is not clear whether the result of [10 is independent of the relaxation time for an arbitrary system.) In the end, we study the asymptotic behavior of the density when the relaxation time $\tau$ tends to zero. Our main results are the following.

Theorem 1. Let $\bar{\rho}>0$, and let $k \in \mathbb{N}$ with $k>d / 2+1$. There exist two constants $\delta>0$ and $C>0$ such that, for all $\tau \in] 0,1]$ and for all initial data $\left(\rho_{0}, \mathbf{m}_{0}\right)$ verifying $\left\|\rho_{0}-\bar{\rho}\right\|_{H^{k}\left(\mathbb{R}^{d}\right)}+\left\|\mathbf{m}_{0}\right\|_{H^{k}\left(\mathbb{R}^{d}\right)} \leq \delta$, there exists a unique global solution $\left(\rho^{\tau}, \mathbf{m}^{\tau}\right)$ to (11) such that $\left(\rho^{\tau}-\bar{\rho}, \mathbf{m}^{\tau}\right) \in \mathcal{C}\left(\mathbb{R}^{+} ; H^{k}\left(\mathbb{R}^{d}\right)\right)$. Moreover, this solution satisfies

$$
\begin{aligned}
\sup _{t \geq 0}\left(\left\|\rho^{\tau}(t)-\bar{\rho}\right\|_{H^{k}\left(\mathbb{R}^{d}\right)}^{2}+\left\|\mathbf{m}^{\tau}(t)\right\|_{H^{k}\left(\mathbb{R}^{d}\right)}^{2}\right)+\frac{1}{\tau} \int_{0}^{+\infty}\left\|\mathbf{m}^{\tau}(s)\right\|_{H^{k}\left(\mathbb{R}^{d}\right)}^{2} d s \\
\leq C\left(\left\|\rho_{0}-\bar{\rho}\right\|_{H^{k}\left(\mathbb{R}^{d}\right)}^{2}+\left\|\mathbf{m}_{0}\right\|_{H^{k}\left(\mathbb{R}^{d}\right)}^{2}\right) .
\end{aligned}
$$

As a consequence, we can study the convergence of the solutions $\rho^{\tau}$ in the strong relaxation limit, that is, when $\tau$ tends to 0 . The initial data are chosen independent of $\tau$, and the convergence is observed on a large time scale. Namely, we shall prove the following statement.

Corollary 1. Let the assumptions of Theorem 1 be fulfilled. Let $\left(\rho^{\tau}, \mathbf{m}^{\tau}\right)$ denote the corresponding solution to (11), and let us set

$$
\varrho^{\tau}(s, x):=\rho^{\tau}(s / \tau, x), \quad \mathbf{v}^{\tau}(s, x):=\mathbf{u}^{\tau}(s / \tau, x) .
$$

Then, $\varrho^{\tau}-\bar{\rho}$ is bounded in $\mathcal{C}\left(\mathbb{R}^{+} ; H^{k}\left(\mathbb{R}^{d}\right)\right)$, and $\left(\varrho^{\tau} \mathbf{v}^{\tau}\right) / \tau$ is bounded in $L^{2}\left(\mathbb{R}^{+} ; H^{k}\left(\mathbb{R}^{d}\right)\right)$. Moreover, if $B_{r}$ denotes the ball of radius $r$ in $\mathbb{R}^{d}$, then for any $0<T, R<\infty$, and for any $0<k^{\prime}<k$, $\left(\varrho^{\tau}\right)$ converges in $\mathcal{C}\left([0, T] ; H^{k^{\prime}}\left(B_{R}\right)\right)$ towards the solution $\varrho \in \mathcal{C}\left(\mathbb{R}^{+} ; \bar{\rho}+H^{k}\left(\mathbb{R}^{d}\right)\right)$ to the heat equation

$$
\begin{cases}\partial_{s} \varrho-a^{2} \Delta_{x} \varrho=0, & s \geq 0, x \in \mathbb{R}^{d}, \\ \left.\varrho\right|_{s=0}=\rho_{0}, & x \in \mathbb{R}^{d} .\end{cases}
$$

\section{UNIFORM WELL-POSEDNESS}

2.1. Preliminary transformations. Recall that system (1) admits the following entropy:

$$
\eta(\rho, \mathbf{m}):=\frac{|\mathbf{m}|^{2}}{2 \rho}+a^{2} \rho \ln \rho
$$

whose flux is

$$
q(\rho, \mathbf{m}):=\frac{|\mathbf{m}|^{2}}{2 \rho^{2}} \mathbf{m}+a^{2}(1+\ln \rho) \mathbf{m} .
$$

It is straightforward to check that the entropy $\eta$ is a strictly convex function of $(\rho, \mathbf{m})$ in the open set $\{(\rho, \mathbf{m}) \in] 0,+\infty\left[\times \mathbb{R}^{d}\right\}$ where (11) is hyperbolic. For smooth solutions to (11), one has the additional balance law

$$
\partial_{t} \eta+\nabla_{x} \cdot q=-\frac{1}{\tau} \rho|\mathbf{u}|^{2} .
$$

We fix once and for all a positive density $\bar{\rho}>0$, and we introduce the entropic variables:

$$
W=\left(\begin{array}{l}
U \\
V
\end{array}\right):=\nabla \eta(\rho, \mathbf{m})-\nabla \eta(\bar{\rho}, 0)=\left(\begin{array}{c}
a^{2} \ln \left(\frac{\rho}{\bar{\rho}}\right)-\frac{1}{2}|\mathbf{u}|^{2} \\
\mathbf{u}
\end{array}\right)
$$


in order to use Godunov's symmetrization. Note that this is a change of variables from the open set $\{(\rho, \mathbf{m}) \in] 0,+\infty\left[\times \mathbb{R}^{d}\right\}$ to the whole space $\mathbb{R}^{1+d}$. After a few simplifications, we show that for smooth solutions $(\rho, \mathbf{m})$ away from vacuum, (1) is equivalent to a quasilinear symmetric hyperbolic system for $W=(U, V)$ :

$$
A_{0}(V) \partial_{t} W+\sum_{j=1}^{d} A_{j}(V) \partial_{x_{j}} W=-\frac{a^{2}}{\tau}\left(\begin{array}{c}
0 \\
V
\end{array}\right),
$$

where $A_{0}(V)$ is a symmetric positive definite matrix, the matrices $A_{j}(V)$ are symmetric, and are defined as follows:

$$
\begin{aligned}
& A_{0}(V):=\left(\begin{array}{cc}
1 & V^{T} \\
V & a^{2} I_{d}+V \otimes V
\end{array}\right), \\
& A_{j}(V):=\left(\begin{array}{cc}
V_{j} & a^{2} e_{j}^{T}+V_{j} V^{T} \\
a^{2} e_{j}+V_{j} V & a^{2}\left(e_{j} \otimes V+V \otimes e_{j}\right)+V_{j}\left(a^{2} I_{d}+V \otimes V\right)
\end{array}\right) .
\end{aligned}
$$

To avoid overloading the notations, we define the new relaxation parameter $\widetilde{\tau}:=$ $\tau / a^{2}$, so that (6) reads

$$
A_{0}(V) \partial_{t} W+\sum_{j=1}^{d} A_{j}(V) \partial_{x_{j}} W=-\frac{1}{\widetilde{\tau}}\left(\begin{array}{c}
0 \\
V
\end{array}\right) .
$$

Observe that the matrices $A_{0}, \ldots, A_{d}$ are independent of $\widetilde{\tau}$, and that they only depend on the component $V$ of the vector $W$. (The sound speed $a$ is fixed once and for all.) This will be extensively used in what follows. In the next paragraph, we are going to show an energy estimate for smooth solutions to (9) that may be defined only locally in time. Such (local in time) smooth solutions exist thanks to Kato's result; see [4] or [5]. This energy estimate will yield global existence of smooth solutions for small enough initial data in the Sobolev space $H^{k}\left(\mathbb{R}^{d}\right), k>1+d / 2$.

2.2. Energy estimates. We first introduce some classical notations. For all integers $\ell$, the Sobolev space $H^{\ell}\left(\mathbb{R}^{d}\right)$ is equipped with the norm

$$
\|f\|_{H^{\ell}\left(\mathbb{R}^{d}\right)}^{2}:=\sum_{\alpha \in \mathbb{N}^{d},|\alpha| \leq \ell} \int_{\mathbb{R}^{d}}\left|\partial_{x}^{\alpha} f(x)\right|^{2} d x \sim \int_{\mathbb{R}^{d}}\left(1+|\xi|^{2}\right)^{\ell}|\widehat{f}(\xi)|^{2} d \xi .
$$

We now fix an integer $k$ such that $k>1+d / 2$. For any positive time $T>0$ and any function $W=(U, V) \in \mathcal{C}\left([0, T] ; H^{k}(\mathbb{R})\right.$ ) (with $U \in \mathbb{R}$ and $V \in \mathbb{R}^{d}$ ), we introduce the energy functional

$$
\begin{aligned}
N_{\widetilde{\tau}}(T)^{2}:=\sup _{0 \leq s \leq T}\|W(s)\|_{H^{k}\left(\mathbb{R}^{d}\right)}^{2} & +\frac{1}{\widetilde{\tau}} \int_{0}^{T}\|V(s)\|_{H^{k}\left(\mathbb{R}^{d}\right)}^{2} d s \\
& +\widetilde{\tau} \int_{0}^{T}\left\|\nabla_{x} W(s)\right\|_{H^{k-1}\left(\mathbb{R}^{d}\right)}^{2} d s .
\end{aligned}
$$

We also define

$$
S(T):=\|W\|_{L^{\infty}\left([0, T] \times \mathbb{R}^{d}\right)}+\left\|\nabla_{x} W\right\|_{L^{\infty}\left([0, T] \times \mathbb{R}^{d}\right)} .
$$

Note that the functional $S$ is independent of the relaxation parameter $\widetilde{\tau}$. Observe also that, since $k>1+d / 2$, Sobolev's imbedding theorem yields the following 
inequalities:

$$
\begin{aligned}
& S(T) \leq \bar{C} N_{\widetilde{\tau}}(T), \quad\|V\|_{L^{2}\left([0, T] ; W^{1, \infty}\left(\mathbb{R}^{d}\right)\right)} \leq \bar{C} \sqrt{\widetilde{\tau}} N_{\widetilde{\tau}}(T), \\
& \left\|\nabla_{x} W\right\|_{L^{2}\left([0, T] ; L^{\infty}\left(\mathbb{R}^{d}\right)\right)} \leq \bar{C} \frac{N_{\widetilde{\tau}}(T)}{\sqrt{\widetilde{\tau}}},
\end{aligned}
$$

for some numerical constant $\bar{C}$.

We are going to prove the following.

Proposition 1. Let $\left.\widetilde{\tau} \in] 0, a^{-2}\right]$, let $T>0$, and assume that $W \in \mathcal{C}\left([0, T] ; H^{k}\left(\mathbb{R}^{d}\right)\right)$ is a solution to (9). There exists an increasing function $C: \mathbb{R}^{+} \rightarrow \mathbb{R}^{+}$, that is independent of $T, \widetilde{\tau}$, and $W$, such that the following inequality holds:

$$
N_{\widetilde{\tau}}(T)^{2} \leq C(S(T))\left(N_{\widetilde{\tau}}(0)^{2}+N_{\widetilde{\tau}}(T)^{3}+N_{\widetilde{\tau}}(T)^{4}\right) .
$$

The proof splits into three steps. One first estimates the $L^{\infty}\left([0, T] ; L^{2}\left(\mathbb{R}^{d}\right)\right)$ norm of $W$ and the $L^{2}\left([0, T] \times \mathbb{R}^{d}\right)$ norm of $V$ by integrating the entropy balance law for the corresponding solution to the isothermal Euler equations. Then one estimates the $L^{\infty}\left([0, T] ; H^{k}\left(\mathbb{R}^{d}\right)\right)$ norm of $W$ and the $L^{2}\left([0, T] ; H^{k}\left(\mathbb{R}^{d}\right)\right)$ norm of $V$, by using the classical energy method for the system (9) (see, e.g., [5]). Special attention is needed to obtain uniform bounds with respect to the relaxation parameter $\widetilde{\tau}$. Eventually, one recovers the $L^{2}\left([0, T] ; H^{k-1}\left(\mathbb{R}^{d}\right)\right)$ estimate of $\nabla_{x} W$ by using the so-called Kawashima condition. (This final step was already achieved in 10, but it is crucial here to check the independence of the constants with respect to $\widetilde{\tau}$.)

In what follows, the constants in the inequalities may depend on the sound speed $a$, but they are always independent of $\tau$, or $\widetilde{\tau}$. We recall that $\tau$ and $\widetilde{\tau}$ only differ by a multiplicative constant. Moreover, $C$ will always denote either a constant, or a nonnegative increasing function of its argument, that may vary from line to line or within the same line.

2.2.1. The $L^{\infty}\left(L^{2}\right)$ estimate of $W$. Let $W \in \mathcal{C}\left([0, T] ; H^{k}\left(\mathbb{R}^{d}\right)\right)$ be a solution to (9) that corresponds (for the original unknown quantities) to a solution $(\rho, \mathbf{m}) \in$ $\mathcal{C}\left([0, T] ; \bar{\rho}+H^{k}\left(\mathbb{R}^{d}\right)\right) \times \mathcal{C}\left([0, T] ; H^{k}\left(\mathbb{R}^{d}\right)\right)$ to the isothermal Euler equations (1), which is bounded away from vacuum.

To obtain the first useful estimate, it is convenient to slightly modify the entropy (44). We set

$$
\widetilde{\eta}(\rho, \mathbf{m}):=\eta(\rho, \mathbf{m})-a^{2} \bar{\rho} \ln \bar{\rho}-a^{2}(1+\ln \bar{\rho})(\rho-\bar{\rho}),
$$

which is still, of course, a strictly convex entropy for the system (11). Its flux is denoted $\widetilde{q}(\rho, \mathbf{m})$. Moreover, the entropy $\widetilde{\eta}$ satisfies

$$
\widetilde{\eta}(\bar{\rho}, 0)=0, \quad \nabla \widetilde{\eta}(\bar{\rho}, 0)=0 .
$$

Actually, $\widetilde{\eta}$ is the sum of the kinetic energy $\left(\rho|\mathbf{u}|^{2}\right) / 2$ and the relative entropy of the density $\rho$ with respect to the constant state $\bar{\rho}$ :

$$
H(\rho \mid \bar{\rho}):=a^{2} \bar{\rho}\left(\frac{\rho}{\bar{\rho}} \ln \left(\frac{\rho}{\bar{\rho}}\right)-\frac{\rho}{\bar{\rho}}+1\right) \geq 0 .
$$

Note that $H(\rho \mid \bar{\rho})$ is a nonnegative quantity and vanishes if, and only if, $\rho=\bar{\rho}$; it is a natural quantity to evaluate how far $\rho$ is from $\bar{\rho}$. 
For the smooth solution $(\rho, \mathbf{m}) \in \mathcal{C}\left([0, T] ; \bar{\rho}+H^{k}\left(\mathbb{R}^{d}\right)\right) \times \mathcal{C}\left([0, T] ; H^{k}\left(\mathbb{R}^{d}\right)\right)$ to (1) we integrate the balance law

$$
\partial_{t} \widetilde{\eta}+\nabla_{x} \cdot \widetilde{q}=-\frac{1}{\tau} \rho|\mathbf{u}|^{2}
$$

over the strip $[0, t] \times \mathbb{R}^{d}$, and we obtain

$$
\left.\int_{\mathbb{R}^{d}} \widetilde{\eta} d x\right|_{0} ^{t}+\frac{1}{\tau} \int_{0}^{t} \int_{\mathbb{R}^{d}} \rho|\mathbf{u}|^{2} d x d s=0 .
$$

Using the convexity properties of $\widetilde{\eta}$, we get

$$
\frac{1}{C}\left(|\rho-\bar{\rho}|^{2}+|\mathbf{u}|^{2}\right) \leq \widetilde{\eta}(\rho, \mathbf{m}) \leq C\left(|\rho-\bar{\rho}|^{2}+|\mathbf{u}|^{2}\right),
$$

where the constant $C$ only depends on $\bar{\rho}$ and the norms $\|\rho-\bar{\rho}\|_{L^{\infty}\left([0, t] \times \mathbb{R}^{d}\right)}$, $\|\mathbf{m}\|_{L^{\infty}\left([0, t] \times \mathbb{R}^{d}\right)}$. To conclude, we use the definition (5), and we thus derive the bounds

$$
\frac{1}{C}|W|^{2} \leq \widetilde{\eta}(\rho, \mathbf{m}) \leq C|W|^{2},
$$

where the constant $C$ only depends on $\bar{\rho}$ and the norm $\|W\|_{L^{\infty}\left([0, t] \times \mathbb{R}^{d}\right)}$, but is independent of the relaxation parameter $\widetilde{\tau}$.

Eventually, we obtain

$$
\forall t \in[0, T], \quad\|W(t)\|_{L^{2}\left(\mathbb{R}^{d}\right)}^{2}+\frac{1}{\widetilde{\tau}} \int_{0}^{t}\|V(s)\|_{L^{2}\left(\mathbb{R}^{d}\right)}^{2} d s \leq C(S(t)) N_{\widetilde{\tau}}(0)^{2} .
$$

2.2.2. The $L^{\infty}\left(H^{k}\right)$ estimate of $W$. Let $\alpha \in \mathbb{N}^{d}$ satisfy $1 \leq|\alpha| \leq k$. We apply the derivative $\partial_{x}^{\alpha}$ to (9), take the scalar product with $\partial_{x}^{\alpha} W$, and integrate over the strip $[0, t] \times \mathbb{R}^{d}$. Integration by parts yields

$$
\begin{aligned}
\left.\frac{1}{2} \int_{\mathbb{R}^{d}} A_{0}(V) \partial_{x}^{\alpha} W \cdot \partial_{x}^{\alpha} W d x\right|_{0} ^{t}+\frac{1}{\widetilde{\tau}} \int_{0}^{t} \int_{\mathbb{R}^{d}}\left|\partial_{x}^{\alpha} V\right|^{2} d x d s \\
\quad=\int_{0}^{t} \int_{\mathbb{R}^{d}}\left[\frac{I_{1}+I_{2}}{2}-\left(I_{3}+I_{4}\right)\right] d x d s
\end{aligned}
$$

where we have set

$$
\begin{array}{ll}
I_{1}:=\left\{\partial_{t} A_{0}(V)\right\} \partial_{x}^{\alpha} W \cdot \partial_{x}^{\alpha} W, & I_{2}:=\sum_{j=1}^{d}\left\{\partial_{x_{j}} A_{j}(V)\right\} \partial_{x}^{\alpha} W \cdot \partial_{x}^{\alpha} W \\
I_{3}:=\left[\partial_{x}^{\alpha}, A_{0}(V)\right] \partial_{t} W \cdot \partial_{x}^{\alpha} W, & I_{4}:=\sum_{j=1}^{d}\left[\partial_{x}^{\alpha}, A_{j}(V)\right] \partial_{x_{j}} W \cdot \partial_{x}^{\alpha} W .
\end{array}
$$

We first estimate the integrals of $I_{1}$ and $I_{2}$. Then we shall estimate the commutators $I_{3}$ and $I_{4}$. Observe that (7) yields

$$
\partial_{t} A_{0}(V)=\left(\begin{array}{cc}
0 & \partial_{t} V^{T} \\
\partial_{t} V & V \otimes \partial_{t} V+\partial_{t} V \otimes V
\end{array}\right) .
$$


Therefore, using (14) and (9), we have

$$
\begin{aligned}
& \int_{0}^{t} \int_{\mathbb{R}^{d}}\left|I_{1}\right| d x d s \leq C(S(t)) \int_{0}^{t} \int_{\mathbb{R}^{d}}\left|\partial_{t} V\right|\left|\partial_{x}^{\alpha} V\right|\left|\partial_{x}^{\alpha} W\right| d x d s \\
& \leq C(S(t)) \int_{0}^{t} \int_{\mathbb{R}^{d}}\left(\left|\nabla_{x} W\right|+\frac{1}{\widetilde{\tau}}|V|\right)\left|\partial_{x}^{\alpha} V\right|\left|\partial_{x}^{\alpha} W\right| d x d s \\
& \leq C(S(t)) \int_{0}^{t}\left(\left\|\nabla_{x} W(s)\right\|_{L^{\infty}\left(\mathbb{R}^{d}\right)}+\frac{1}{\widetilde{\tau}}\|V(s)\|_{L^{\infty}\left(\mathbb{R}^{d}\right)}\right) \\
& \times\left\|\partial_{x}^{\alpha} V(s)\right\|_{L^{2}\left(\mathbb{R}^{d}\right)}\left\|\partial_{x}^{\alpha} W(s)\right\|_{L^{2}\left(\mathbb{R}^{d}\right)} d s .
\end{aligned}
$$

To conclude, we use the obvious bound $\left\|\partial_{x}^{\alpha} W(s)\right\|_{L^{2}\left(\mathbb{R}^{d}\right)} \leq N_{\widetilde{\tau}}(t)$, as well as Sobolev's imbedding $H^{k-1}\left(\mathbb{R}^{d}\right) \subset L^{\infty}\left(\mathbb{R}^{d}\right)$ and Cauchy-Schwarz' inequality. We get

$$
\int_{0}^{t} \int_{\mathbb{R}^{d}}\left|I_{1}\right| d x d s \leq C(S(t)) N_{\widetilde{\tau}}(t)^{3} .
$$

The estimate of $I_{2}$ is rather similar. From (14), we get

$$
\begin{aligned}
\int_{0}^{t} \int_{\mathbb{R}^{d}}\left|I_{2}\right| d x d s \leq C(S(t)) & \int_{0}^{t} \int_{\mathbb{R}^{d}}\left|\nabla_{x} V\right|\left|\partial_{x}^{\alpha} W\right|^{2} d x d s \\
& \leq C(S(t)) \int_{0}^{t}\left\|\nabla_{x} V(s)\right\|_{L^{\infty}\left(\mathbb{R}^{d}\right)}\left\|\partial_{x}^{\alpha} W(s)\right\|_{L^{2}\left(\mathbb{R}^{d}\right)}^{2} d s .
\end{aligned}
$$

Again, we use the bound $\left\|\partial_{x}^{\alpha} W(s)\right\|_{L^{2}\left(\mathbb{R}^{d}\right)} \leq N_{\widetilde{\tau}}(t)$, Sobolev's imbedding, and Cauchy-Schwarz' inequality. We end up with

$$
\int_{0}^{t} \int_{\mathbb{R}^{d}}\left|I_{2}\right| d x d s \leq C(S(t)) N_{\widetilde{\tau}}(t)^{3} .
$$

To estimate $I_{3}$, we first apply Cauchy-Schwarz' inequality:

$$
\int_{0}^{t} \int_{\mathbb{R}^{d}}\left|I_{3}\right| d x d s \leq \int_{0}^{t}\left\|\left[\partial_{x}^{\alpha}, A_{0}(V)\right] \partial_{t} W(s)\right\|_{L^{2}\left(\mathbb{R}^{d}\right)}\left\|\partial_{x}^{\alpha} W(s)\right\|_{L^{2}\left(\mathbb{R}^{d}\right)} d s,
$$

and we use the classical tame estimate for a commutator; see, e.g., [5, page 43]. For a fixed time $s \in[0, t]$, we obtain

$$
\begin{aligned}
\left\|\left[\partial_{x}^{\alpha}, A_{0}(V)\right] \partial_{t} W(s)\right\|_{L^{2}\left(\mathbb{R}^{d}\right)} \leq & C\left(\left\|\partial_{t} W(s)\right\|_{L^{\infty}\left(\mathbb{R}^{d}\right)}\left\|\nabla_{x} A_{0}(V)(s)\right\|_{H^{k-1}\left(\mathbb{R}^{d}\right)}\right. \\
& \left.+\left\|\partial_{t} W(s)\right\|_{H^{k-1}\left(\mathbb{R}^{d}\right)}\left\|\nabla_{x} A_{0}(V)(s)\right\|_{L^{\infty}\left(\mathbb{R}^{d}\right)}\right) .
\end{aligned}
$$

Using (9) to obtain the expression of $\partial_{t} W$ in terms of $\nabla_{x} W$ and $V$, we have, on the one hand

$$
\left\|\partial_{t} W(s)\right\|_{L^{\infty}\left(\mathbb{R}^{d}\right)} \leq C(S(t))\left(\left\|\nabla_{x} W(s)\right\|_{L^{\infty}\left(\mathbb{R}^{d}\right)}+\frac{1}{\widetilde{\tau}}\|V(s)\|_{L^{\infty}\left(\mathbb{R}^{d}\right)}\right),
$$

while, on the other hand, the classical tame estimate for composed functions (see, e.g., [1, page 101]) yields

$$
\left\|\partial_{t} W(s)\right\|_{H^{k-1}\left(\mathbb{R}^{d}\right)} \leq C(S(t))\left(\|W(s)\|_{H^{k}\left(\mathbb{R}^{d}\right)}+\frac{1}{\widetilde{\tau}}\|V(s)\|_{H^{k-1}\left(\mathbb{R}^{d}\right)}\right) .
$$

The tame estimate of composed functions also enables us to derive the bound

$$
\left\|\nabla_{x} A_{0}(V)(s)\right\|_{H^{k-1}\left(\mathbb{R}^{d}\right)} \leq C(S(t))\|V(s)\|_{H^{k}\left(\mathbb{R}^{d}\right)} .
$$


Using the three previous estimates in (19), we end up with

$$
\begin{aligned}
& \left\|\left[\partial_{x}^{\alpha}, A_{0}(V)\right] \partial_{t} W(s)\right\|_{L^{2}\left(\mathbb{R}^{d}\right)} \\
& \quad \leq C(S(t))\|V(s)\|_{H^{k}\left(\mathbb{R}^{d}\right)}\left(\|W(s)\|_{H^{k}\left(\mathbb{R}^{d}\right)}+\frac{1}{\widetilde{\tau}}\|V(s)\|_{H^{k}\left(\mathbb{R}^{d}\right)}\right) .
\end{aligned}
$$

With this estimate for the commutator, (18) yields

$$
\begin{array}{r}
\int_{0}^{t} \int_{\mathbb{R}^{d}}\left|I_{3}\right| d x d s \leq C(S(t)) \int_{0}^{t}\left\|\nabla_{x} W(s)\right\|_{H^{k-1}\left(\mathbb{R}^{d}\right)}\|V(s)\|_{H^{k}\left(\mathbb{R}^{d}\right)} \\
\left(\|W(s)\|_{H^{k}\left(\mathbb{R}^{d}\right)}+\frac{1}{\widetilde{\tau}}\|V(\tau)\|_{H^{k}\left(\mathbb{R}^{d}\right)}\right) d s .
\end{array}
$$

To conclude, we use either the bound $\|W(s)\|_{H^{k}\left(\mathbb{R}^{d}\right)} \leq N_{\widetilde{\tau}}(t)$, or the bound $\left\|\nabla_{x} W(s)\right\|_{H^{k-1}\left(\mathbb{R}^{d}\right)} \leq N_{\widetilde{\tau}}(t)$, and Cauchy-Schwarz' inequality. We have thus derived

$$
\int_{0}^{t} \int_{\mathbb{R}^{d}}\left|I_{3}\right| d x d s \leq C(S(t)) N_{\widetilde{\tau}}(t)^{3} .
$$

The estimate of $I_{4}$ is simpler, since $I_{4}$ only involves spatial derivatives; see (15). We proceed as above, and we obtain the estimate

$$
\int_{0}^{t} \int_{\mathbb{R}^{d}}\left|I_{4}\right| d x d s \leq C(S(t)) N_{\widetilde{\tau}}(t)^{3} .
$$

The left-hand side of (13) is easily estimated from below, and the right-hand side is estimated by (16), (17), (20), (21). Summing over the multi-integers $\alpha$, and using (12), we obtain the $L^{\infty}\left([0, T] ; H^{k}\left(\mathbb{R}^{d}\right)\right)$ uniform estimate:

$$
\forall t \in[0, T], \quad\|W(t)\|_{H^{k}\left(\mathbb{R}^{d}\right)}^{2}+\frac{1}{\widetilde{\tau}} \int_{0}^{t}\|V(s)\|_{H^{k}\left(\mathbb{R}^{d}\right)}^{2} d s \leq C(S(t))\left(N_{\widetilde{\tau}}(0)^{2}+N_{\widetilde{\tau}}(t)^{3}\right) .
$$

2.2.3. The $L^{2}\left(H^{k-1}\right)$ estimate of $\nabla_{x} W$. In this paragraph, we follow the method developed in [10. Recall that the matrices $A_{0}, \ldots, A_{d}$ are defined by (7) and (8). We begin with the following elementary result.

Lemma 1. For $\xi \in \mathbb{R}^{d}, \xi \neq 0$, we define a real matrix $K(\xi)$ in the following way:

$$
K(\xi):=\left(\begin{array}{cc}
0 & \frac{1}{a^{2}} \frac{\xi^{T}}{|\xi|} \\
-\frac{\xi}{|\xi|} & 0
\end{array}\right) .
$$

Then $K(\xi) A_{0}(0)$ is skew-symmetric, and

$$
K(\xi) \sum_{j=1}^{d} \xi_{j} A_{j}(0)=\left(\begin{array}{cc}
|\xi| & 0 \\
0 & -a^{2} \frac{\xi \otimes \xi}{|\xi|}
\end{array}\right) .
$$

We rewrite (9) as follows:

$$
A_{0}(0) \partial_{t} W+\sum_{j=1}^{d} A_{j}(0) \partial_{x_{j}} W=-\frac{1}{\widetilde{\tau}}\left(\begin{array}{c}
0 \\
V
\end{array}\right)+h,
$$


where we have set

$$
\begin{aligned}
h:=\frac{1}{\widetilde{\tau}}\{I- & \left.A_{0}(0) A_{0}(V)^{-1}\right\}\left(\begin{array}{c}
0 \\
V
\end{array}\right) \\
& +\sum_{j=1}^{d}\left\{A_{j}(0)-A_{j}(V)-\left(A_{0}(0)-A_{0}(V)\right) A_{0}(V)^{-1} A_{j}(V)\right\} \partial_{x_{j}} W .
\end{aligned}
$$

Since $k-1>d / 2$, the Sobolev space $H^{k-1}\left(\mathbb{R}^{d}\right)$ is an algebra. Therefore, we have

$$
\begin{aligned}
\forall s \in[0, t], \quad & \|h(s)\|_{H^{k-1}\left(\mathbb{R}^{d}\right)} \leq C(S(t)) \\
& \left(\frac{1}{\widetilde{\tau}}\|V(s)\|_{H^{k-1}\left(\mathbb{R}^{d}\right)}^{2}+\|V(s)\|_{H^{k-1}\left(\mathbb{R}^{d}\right)}\left\|\nabla_{x} W(s)\right\|_{H^{k-1}\left(\mathbb{R}^{d}\right)}\right),
\end{aligned}
$$

for a suitable increasing function $C$ that is independent of the relaxation parameter $\widetilde{\tau}$.

We take the Fourier transform (in the space variable $x$ ) of (23), multiply by $-i \widetilde{\tau}(\widehat{W})^{*} K(\xi)$, and then compute the real part of each term in the equality. The notation $X^{*}$ stands for the transposed conjugate of a vector $X \in \mathbb{C}^{q}, q \in \mathbb{N}$. Using the expression of the matrix $K(\xi)$ (see Lemma 1) we obtain

$$
\begin{aligned}
\widetilde{\tau} \operatorname{Im}\left((\widehat{W})^{*} K(\xi) A_{0}(0) \frac{d \widehat{W}}{d t}\right)+\widetilde{\tau}(\widehat{W})^{*} K(\xi)\left(\sum_{j=1}^{d} \xi_{j} A_{j}(0)\right) \widehat{W} \\
=-\frac{1}{a^{2}} \operatorname{Im}\left((\widehat{U})^{*} \frac{\xi^{T} \widehat{V}}{|\xi|}\right)+\widetilde{\tau} \operatorname{Im}\left((\widehat{W})^{*} K(\xi) \widehat{h}\right) .
\end{aligned}
$$

The left-hand side of (25) is simplified by observing that the skew-symmetry of $K(\xi) A_{0}(0)$ (see Lemma 11) implies the relation

$$
\operatorname{Im}\left((\widehat{W})^{*} K(\xi) A_{0}(0) \frac{d \widehat{W}}{d t}\right)=\operatorname{Re} \frac{d}{d t}\left((\widehat{W})^{*} K(\xi) A_{0}(0) \widehat{W}\right) .
$$

Using Lemma 1 it is now straightforward to derive a lower bound for the left-hand side of (25). Indeed, we have

$$
\begin{aligned}
\widetilde{\tau} \operatorname{Im}\left((\widehat{W})^{*} K(\xi) A_{0}(0) \frac{d \widehat{W}}{d t}\right)+\widetilde{\tau}(\widehat{W})^{*} K(\xi)\left(\sum_{j=1}^{d} \xi_{j} A_{j}(0)\right) \widehat{W} \\
\geq \widetilde{\tau} \operatorname{Re} \frac{d}{d t}\left((\widehat{W})^{*} K(\xi) A_{0}(0) \widehat{W}\right)+\widetilde{\tau}|\xi||\widehat{W}|^{2}-C \widetilde{\tau}|\xi||\widehat{V}|^{2}
\end{aligned}
$$

The right-hand side of (25) can be estimated thanks to Young's inequality, and thanks to a uniform bound for the matrices $K(\xi)$ (see Lemma 1). For $\xi \neq 0$, we obtain

$-\frac{1}{a^{2}} \operatorname{Im}\left((\widehat{U})^{*} \frac{\xi^{T} \widehat{V}}{|\xi|}\right)+\widetilde{\tau} \operatorname{Im}\left((\widehat{W})^{*} K(\xi) \widehat{h}\right) \leq \frac{\widetilde{\tau}}{2}|\xi||\widehat{W}|^{2}+\frac{C}{\widetilde{\tau}|\xi|}|\widehat{V}|^{2}+\frac{C \widetilde{\tau}}{|\xi|}|\widehat{h}|^{2}$.

Combining (26) and (27) in (25) yields

(28) $\frac{\widetilde{\tau}}{2}|\xi||\widehat{W}|^{2} \leq \frac{C}{\widetilde{\tau}}\left(\frac{1}{|\xi|}+|\xi|\right)|\widehat{V}|^{2}+\frac{C \widetilde{\tau}}{|\xi|}|\widehat{h}|^{2}-\widetilde{\tau} \operatorname{Re} \frac{d}{d t}\left((\widehat{W})^{*} K(\xi) A_{0}(0) \widehat{W}\right)$. 
We consider an integer $\ell$ such that $1 \leq \ell \leq k$. We multiply (28) by $|\xi|^{2 \ell-1}$, integrate over $[0, t] \times \mathbb{R}^{d}$, and then use Plancherel's Theorem:

$$
\begin{aligned}
\widetilde{\tau} \int_{0}^{t} \int_{\mathbb{R}^{d}} \sum_{|\alpha|=\ell}\left|\partial_{x}^{\alpha} W\right|^{2} d x d s \leq-\left.C \widetilde{\tau} \operatorname{Re} \int_{\mathbb{R}^{d}}|\xi|^{2 \ell-1}\left((\widehat{W})^{*} K(\xi) A_{0}(0) \widehat{W}\right) d \xi\right|_{0} ^{t} \\
+\frac{C}{\widetilde{\tau}} \int_{0}^{t}\|V(s)\|_{H^{\ell}\left(\mathbb{R}^{d}\right)}^{2} d s+C \widetilde{\tau} \int_{0}^{t}\|h(s)\|_{H^{\ell-1}\left(\mathbb{R}^{d}\right)}^{2} d s
\end{aligned}
$$

The matrices $K(\xi)$ are uniformly bounded when $\xi \in \mathbb{R}^{d}, \xi \neq 0$, and we thus have

$$
\begin{aligned}
& -\left.C \widetilde{\tau} \operatorname{Re} \int_{\mathbb{R}^{d}}|\xi|^{2 \ell-1}\left((\widehat{W})^{*} K(\xi) A_{0}(0) \widehat{W}\right) d \xi\right|_{0} ^{t} \\
& \leq C \widetilde{\tau}\left(\int_{\mathbb{R}^{d}}\left(1+|\xi|^{2}\right)^{\ell}|\widehat{W}(t)|^{2} d \xi+\int_{\mathbb{R}^{d}}\left(1+|\xi|^{2}\right)^{\ell}|\widehat{W}(0)|^{2} d \xi\right) \\
& \leq C \widetilde{\tau}\left(\|W(t)\|_{H^{\ell}\left(\mathbb{R}^{d}\right)}^{2}+\|W(0)\|_{H^{\ell}\left(\mathbb{R}^{d}\right)}^{2}\right) .
\end{aligned}
$$

Using (22), (29) thus leads to

$$
\begin{aligned}
& \widetilde{\tau} \int_{0}^{t} \sum_{|\alpha|=\ell}\left\|\partial_{x}^{\alpha} W(s)\right\|_{L^{2}\left(\mathbb{R}^{d}\right)}^{2} d s \\
& \leq C(S(t))\left(N_{\widetilde{\tau}}(0)^{2}+N_{\widetilde{\tau}}(t)^{3}\right)+C \widetilde{\tau} \int_{0}^{t}\|h(s)\|_{H^{k-1}\left(\mathbb{R}^{d}\right)}^{2} d s .
\end{aligned}
$$

To conclude, we use the $H^{k-1}\left(\mathbb{R}^{d}\right)$ estimate of the source term $h$; see (24). We obtain

$$
\begin{aligned}
& \widetilde{\tau} \int_{0}^{t}\|h(s)\|_{H^{k-1}\left(\mathbb{R}^{d}\right)}^{2} d s \leq C(S(t)) \int_{0}^{t} \frac{1}{\widetilde{\tau}}\|V(s)\|_{H^{k-1}\left(\mathbb{R}^{d}\right)}^{4} \\
+ & \|V(s)\|_{H^{k-1}\left(\mathbb{R}^{d}\right)}^{3}\left\|\nabla_{x} W(s)\right\|_{H^{k-1}\left(\mathbb{R}^{d}\right)}+\widetilde{\tau}\|V(s)\|_{H^{k-1}\left(\mathbb{R}^{d}\right)}^{2}\left\|\nabla_{x} W(s)\right\|_{H^{k-1}\left(\mathbb{R}^{d}\right)}^{2} d s .
\end{aligned}
$$

We use the bound $\|V(s)\|_{H^{k-1}\left(\mathbb{R}^{d}\right)} \leq N_{\widetilde{\tau}}(t)$ and Cauchy-Schwarz' inequality. In the end, we are led to the estimate

$$
\widetilde{\tau} \int_{0}^{t}\|h(s)\|_{H^{k-1}\left(\mathbb{R}^{d}\right)}^{2} d s \leq C(S(t)) N_{\widetilde{\tau}}(t)^{4} .
$$

Using this final estimate in (30), and summing over $\ell=1, \ldots, k-1$, we obtain the estimate of $\nabla_{x} W$ in $L^{2}\left([0, T] ; H^{k-1}\left(\mathbb{R}^{d}\right)\right)$. Together with (22), this completes the proof of Proposition 1 .

2.3. End of the proof of Theorem 1, To conclude the proof, we follow [7. Using Proposition 1, we first deduce that there exists a numerical constant $C_{0} \geq 1$ such that, if $W \in \mathcal{C}\left([0, T] ; H^{k}\left(\mathbb{R}^{d}\right)\right)$ is a solution to (9) that satisfies $N_{\widetilde{\tau}}(T) \leq 1$, then $W$ also satisfies

$$
N_{\widetilde{\tau}}(T)^{2} \leq C_{0}\left(N_{\widetilde{\tau}}(0)^{2}+N_{\widetilde{\tau}}(T)^{3}\right) .
$$

The constant $C_{0}$ is, of course, independent of $\widetilde{\tau}$. Consequently, if $W$ is a smooth solution on a time interval $[0, T]$ that satisfies $N_{\widetilde{\tau}}(T) \leq 1 /\left(2 C_{0}\right)$, then $W$ also satisfies

$$
N_{\widetilde{\tau}}(T) \leq \sqrt{2 C_{0}} N_{\widetilde{\tau}}(0) .
$$


Before going on, we observe that $N_{\widetilde{\tau}}(0)$ is independent of $\widetilde{\tau}$ (see (10)) since $N_{\widetilde{\tau}}(0)$ is nothing but the norm of the initial data $W(0)$ in $H^{k}\left(\mathbb{R}^{d}\right)$.

Consider an initial condition $W(0) \in H^{k}\left(\mathbb{R}^{d}\right)$ such that

$$
\|W(0)\|_{H^{k}\left(\mathbb{R}^{d}\right)} \leq 1 /\left[2\left(2 C_{0}\right)^{3 / 2}\right] .
$$

Assume that the corresponding smooth solution $W$ to (9) is not global, and thus blows up in finite time, say at time $T_{*}>0$. This means that for some positive time $T_{0}$, one has

$$
\left.N_{\widetilde{\tau}}\left(T_{0}\right)=\frac{1}{4 C_{0}}>N_{\widetilde{\tau}}(0) \quad \text { and } \quad \forall t \in\right] T_{0}, T_{*}\left[, \quad N_{\widetilde{\tau}}(t)>\frac{1}{4 C_{0}} .\right.
$$

Since $N_{\widetilde{\tau}}\left(T_{0}\right)<1 /\left(2 C_{0}\right)$, there exists a time $\left.T_{1} \in\right] T_{0}, T_{*}\left[\right.$ such that $N_{\widetilde{\tau}}\left(T_{1}\right) \leq$ $1 /\left(2 C_{0}\right)$, and, applying (31), we obtain

$$
N_{\widetilde{\tau}}\left(T_{1}\right) \leq \sqrt{2 C_{0}} N_{\widetilde{\tau}}(0) \leq \frac{\sqrt{2 C_{0}}}{2\left(2 C_{0}\right)^{3 / 2}} \leq \frac{1}{4 C_{0}} .
$$

We are led to a contradiction. The smooth solution is thus global in time for small enough initial data. The key point is that the smallness of the initial data is independent of $\widetilde{\tau}$. Moreover, when $\|W(0)\|_{H^{k}\left(\mathbb{R}^{d}\right)} \leq 1 /\left[2\left(2 C_{0}\right)^{3 / 2}\right]$, we have the (global in time) uniform estimate

$$
\forall t \geq 0, \quad N_{\widetilde{\tau}}(t) \leq \min \left(\frac{1}{2 C_{0}}, \sqrt{2 C_{0}} N_{\widetilde{\tau}}(0)\right) .
$$

It remains to convert the result for the system (9) into a result for the system (11). Using (5), we compute

$$
\rho-\bar{\rho}=\bar{\rho}\left[\exp \left(U+\frac{1}{2}|V|^{2}\right)-1\right], \quad \mathbf{u}=V .
$$

Consequently, there exists a number $\delta>0$ that is independent of $\widetilde{\tau}$ such that, if

$$
\left\|\rho_{0}-\bar{\rho}\right\|_{H^{k}\left(\mathbb{R}^{d}\right)}+\left\|\mathbf{m}_{0}\right\|_{H^{k}\left(\mathbb{R}^{d}\right)} \leq \delta,
$$

then (11) has a global smooth solution $\left(\rho^{\tau}, \mathbf{m}^{\tau}\right)$, with initial data $\left(\rho_{0}, \mathbf{m}_{0}\right)$, and that satisfies the global uniform estimate

$$
\begin{aligned}
\sup _{t \geq 0}\left(\left\|\rho^{\tau}(t)-\bar{\rho}\right\|_{H^{k}\left(\mathbb{R}^{d}\right)}^{2}+\left\|\mathbf{m}^{\tau}(t)\right\|_{H^{k}\left(\mathbb{R}^{d}\right)}^{2}\right) & +\frac{1}{\tau} \int_{0}^{+\infty}\left\|\mathbf{m}^{\tau}(s)\right\|_{H^{k}\left(\mathbb{R}^{d}\right)}^{2} d s \\
& \leq C\left(\left\|\rho_{0}-\bar{\rho}\right\|_{H^{k}\left(\mathbb{R}^{d}\right)}^{2}+\left\|\mathbf{m}_{0}\right\|_{H^{k}\left(\mathbb{R}^{d}\right)}^{2}\right),
\end{aligned}
$$

for a suitable numerical constant $C$ that is independent of the relaxation time $\tau$ (but that depends on the fixed reference density $\bar{\rho}$, on the sound speed $a$, and on the radius $\delta$ of the ball in $\left.H^{k}\left(\mathbb{R}^{d}\right)\right)$.

\section{Convergence towards the heAt EQUATION}

Let $\left(\rho^{\tau}, \mathbf{m}^{\tau}\right)$ stand for the solution to (1) obtained in Theorem 11, for some given initial data $\left(\rho_{0}, \mathbf{m}_{0}\right)$ that are independent of $\tau$. Performing the limit $\tau \rightarrow 0$ in (1) directly is not relevant at all, since it would simply lead to the trivial limit equation $\mathbf{m}=0, \partial_{t} \rho=0$. Interesting phenomena can be observed by looking at a large time scale. Indeed, we change the time variable by considering an "OO$(1 / \tau)$ time scale":

$$
\varrho^{\tau}(s, x):=\rho^{\tau}(s / \tau, x), \quad \mathbf{v}^{\tau}(s, x):=\mathbf{u}^{\tau}(s / \tau, x) .
$$


(Observe that the new "time" variable $s$ is actually homogeneous to the square of a time, since $\tau$ is homogeneous to a time.) The new unknowns satisfy the following system:

$$
\begin{array}{r}
\partial_{s} \varrho^{\tau}+\nabla_{x} \cdot\left(\frac{\varrho^{\tau} \mathbf{v}^{\tau}}{\tau}\right)=0, \\
\tau^{2} \partial_{s}\left(\frac{\varrho^{\tau} \mathbf{v}^{\tau}}{\tau}\right)+\tau^{2} \nabla_{x} \cdot\left(\frac{\varrho^{\tau} \mathbf{v}^{\tau} \otimes \mathbf{v}^{\tau}}{\tau^{2}}\right)=-\frac{\varrho^{\tau} \mathbf{v}^{\tau}}{\tau}-a^{2} \nabla_{x} \varrho^{\tau} .
\end{array}
$$

We deduce directly from (2) that both quantities $\sup _{s \geq 0}\left\|\varrho^{\tau}(s)-\bar{\rho}\right\|_{H^{k}\left(\mathbb{R}^{d}\right)}$ and

$$
\frac{1}{\tau} \int_{0}^{\infty}\left\|\mathbf{m}^{\tau}(t)\right\|_{H^{k}\left(\mathbb{R}^{d}\right)}^{2} d t=\frac{1}{\tau^{2}} \int_{0}^{\infty}\left\|\varrho^{\tau} \mathbf{v}^{\tau}(\sigma)\right\|_{H^{k}\left(\mathbb{R}^{d}\right)}^{2} d \sigma
$$

are bounded uniformly with respect to $\tau>0$. Moreover, we know that the density $\varrho^{\tau}$ is uniformly bounded from above and from below. This allows us to readily obtain the heat equation as $\tau$ tends to 0 . Indeed, the left-hand side of (33) reads as $\tau^{2} \times$ the time derivative of a quantity that is bounded in $L^{2}\left(\mathbb{R}^{+} \times \mathbb{R}^{d}\right)$, plus $\tau^{2} \times$ the space derivative of a quantity that is bounded in $L^{1}\left(\mathbb{R}^{+} \times \mathbb{R}^{d}\right)$. Hence, as $\tau$ goes to 0 , we are led to

$$
-\frac{\varrho^{\tau} \mathbf{v}^{\tau}}{\tau}-a^{2} \nabla_{x} \varrho^{\tau} \rightarrow 0 \quad \text { in } \mathcal{D}^{\prime}\left(\mathbb{R}^{+} \times \mathbb{R}^{d}\right)
$$

Inserting this information into (32), we get

$$
\partial_{s} \varrho^{\tau}-a^{2} \Delta_{x} \varrho^{\tau} \rightarrow 0 \quad \text { in } \mathcal{D}^{\prime}\left(\mathbb{R}^{+} \times \mathbb{R}^{d}\right) \text { as } \tau \rightarrow 0 .
$$

Let $\varrho \in \mathcal{C}\left(\mathbb{R}^{+} ; \bar{\rho}+H^{k}\left(\mathbb{R}^{d}\right)\right)$ denote the solution to the heat equation (3) with initial data $\rho_{0} \in \bar{\rho}+H^{k}\left(\mathbb{R}^{d}\right)$. We want to make precise the convergence of the sequence $\left(\varrho^{\tau}\right)$ towards $\varrho$. First we are going to prove that $\left(\varrho^{\tau}\right)$ tends towards $\varrho$ in the sense of distributions in $\mathbb{R}^{+} \times \mathbb{R}^{d}$. Then we are going to show the convergence stated in Corollary 1

Let $T>0$. We first note that (32) implies that $\left(\partial_{s} \varrho^{\tau}\right)$ is bounded in the space $L^{2}\left(\mathbb{R}^{+} ; H^{k-1}\left(\mathbb{R}^{d}\right)\right)$. Moreover, $\left(\varrho^{\tau}-\bar{\rho}\right)$ is bounded in $\mathcal{C}\left(\mathbb{R}^{+} ; H^{k}\left(\mathbb{R}^{d}\right)\right)$, and is thus also bounded in $L^{2}\left(0, T ; H^{k-1}\left(\mathbb{R}^{d}\right)\right)$. In particular, we deduce that there exists a subsequence $\left(\tau_{n}\right)$ that tends to 0 , and there exists a function $\widetilde{\varrho}$ such that

$\widetilde{\varrho}-\bar{\rho} \in H^{1}\left(0, T ; H^{k-1}\left(\mathbb{R}^{d}\right)\right)$ and $\varrho^{\tau_{n}}-\bar{\rho} \rightarrow \widetilde{\varrho}-\bar{\rho}$ weakly in $H^{1}\left(0, T ; H^{k-1}\left(\mathbb{R}^{d}\right)\right)$. We have $\widetilde{\varrho} \in \mathcal{C}\left([0, T] ; \bar{\rho}+H^{k-1}\left(\mathbb{R}^{d}\right)\right)$, and since $\left.\varrho^{\tau_{n}}\right|_{s=0}=\rho_{0}$ for all $n$, we obtain $\left.\widetilde{\varrho}\right|_{s=0}=\rho_{0}$. To prove this, we observe that, for all points $y \in \mathbb{R}^{d}$, the linear form $\delta_{(s, x)=(0, y)}$ is well defined and continuous on $H^{1}\left(0, T ; H^{k-1}\left(\mathbb{R}^{d}\right)\right)$. Therefore, for all point $y \in \mathbb{R}^{d}$, we have

$$
\varrho^{\tau_{n}}(0, y)=\rho_{0}(y) \longrightarrow \widetilde{\varrho}(0, y) \text { as } n \rightarrow+\infty .
$$

Now, we note that (34) implies that $\widetilde{\varrho} \in \mathcal{C}\left([0, T] ; \bar{\rho}+H^{k-1}\left(\mathbb{R}^{d}\right)\right)$ is a solution to the heat equation with initial data $\rho_{0}$. Hence $\widetilde{\varrho}=\varrho \in \mathcal{C}\left([0, T] ; \bar{\rho}+H^{k}\left(\mathbb{R}^{d}\right)\right)$. Now, a classical argument shows that the whole sequence $\left(\varrho^{\tau}-\varrho\right)$ converges towards zero for the weak topology of $H^{1}\left(0, T ; H^{k-1}\left(\mathbb{R}^{d}\right)\right)$. In particular, we obtain

$$
\varrho^{\tau} \rightarrow \varrho \quad \text { in } \mathcal{D}^{\prime}\left(\mathbb{R}^{+} \times \mathbb{R}^{d}\right) \text { as } \tau \rightarrow 0 .
$$

Let $T>0$, and let $0<k^{\prime}<k$. We have already noted that, thanks to (32), $\left(\partial_{s} \varrho^{\tau}\right)$ is bounded in $L^{2}\left(\mathbb{R}^{+}, H^{k-1}\left(\mathbb{R}^{d}\right)\right)$. Moreover, for any $R>0,\left(\varrho^{\tau}\right)$ is bounded in $\mathcal{C}\left(\mathbb{R}^{+} ; H^{k}\left(B_{R}\right)\right) \mathbb{1}$ Hence, we can apply the compactness result of [9, Corollary

\footnotetext{
${ }^{1}$ Recall that $B_{R}$ denotes the ball of radius $R$ in $\mathbb{R}^{d}$.
} 
4]; we deduce that $\left(\varrho^{\tau}\right)$ is relatively compact in $\mathcal{C}\left([0, T] ; H^{k^{\prime}}\left(B_{R}\right)\right)$ for any $R>0$. Note that any limit value of a subsequence of $\left(\varrho^{\tau}\right)$ in $\mathcal{C}\left([0, T] ; H^{k^{\prime}}\left(B_{R}\right)\right)$ is also a limit in the space of distributions $\mathcal{D}^{\prime}(] 0, T\left[\times B_{R}\right)$. Consequently, any limit value of a subsequence of $\left(\varrho^{\tau}\right)$ in the space $\mathcal{C}\left([0, T] ; H^{k^{\prime}}\left(B_{R}\right)\right)$ is equal to $\varrho$. We have thus obtained the convergence of the whole sequence $\left(\varrho^{\tau}\right)$ in the space $\mathcal{C}\left([0, T] ; H^{k^{\prime}}\left(B_{R}\right)\right)$ :

$$
\forall R>0, \quad \varrho^{\tau} \longrightarrow \varrho \quad \text { in } \mathcal{C}\left([0, T] ; H^{k^{\prime}}\left(B_{R}\right)\right) .
$$

The proof of Corollary 1 is complete.

\section{REFERENCES}

1. S. Alinhac, P. Gérard, Opérateurs pseudo-différentiels et théorème de Nash-Moser, InterEditions, 1991. MR1172111 (93g:35001)

2. B. Hanouzet, R. Natalini, Global existence of smooth solutions for partially dissipative hyperbolic systems with a convex entropy, Arch. Ration. Mech. Anal. 169 (2003), no. 2, 89-117. MR 2005637 (2004h:35135)

3. S. Junca, M. Rascle, Strong relaxation of the isothermal Euler system to the heat equation, Z. Angew. Math. Phys. 53 (2002), no. 2, 239-264. MR.1900673(2003d:35215)

4. T. Kato, The Cauchy problem for quasi-linear symmetric hyperbolic systems, Arch. Rational Mech. Anal. 58 (1975), no. 3, 181-205. MR0390516 (52:11341)

5. A. Majda, Compressible fluid flow and systems of conservation laws in several space variables, Springer-Verlag, 1984. MR0748308 (85e:35077)

6. P. Marcati, A. Milani, The one-dimensional Darcy's law as the limit of a compressible Euler flow, J. Differential Equations 84 (1990), no. 1, 129-147. MR1042662 (91i:35156)

7. T. Nishida, Nonlinear hyperbolic equations and related topics in fluid dynamics, Département de Mathématique, Université de Paris-Sud, Orsay, 1978. MR0481578 (58:1690)

8. T. C. Sideris, B. Thomases, and D. Wang, Long time behavior of solutions to the $3 D$ compressible Euler equations with damping, Comm. Partial Differential Equations 28 (2003), no. 3-4, 795-816. MR1978315 (2004d:35208)

9. J. Simon, Compact sets in the space $L^{p}(0, T ; B)$, Ann. Mat. Pura Appl. (4) 146 (1987), 65-96. MR 0916688 (89c:46055)

10. W.-A. Yong, Entropy and global existence for hyperbolic balance laws, Arch. Ration. Mech. Anal. 172 (2004), no. 2, 247-266. MR2058165 (2005c:35195)

Team SimpaF-INRIA Futurs, CNRS \& Université Lille 1, Laboratoire Paul Painlevé, UMR CNRS 8524, Cité Scientifique, 59655 Villeneuve D'Asce Cedex, France

E-mail address: jfcoulom@math.univ-lille1.fr

Team SimpaF-INRIA Futurs, CNRS \& Université Lille 1, Laboratoire Paul Painlevé, UMR CNRS 8524, Cité Scientifique, 59655 Villeneuve D’Ascq Cedex, France

E-mail address: thierry.goudon@math.univ-lille1.fr 